\title{
Utilization of Channel Reciprocity in Advanced MIMO System
}

\author{
Qiubin Gao, Fei Qin, Shaohui Sun \\ System and Standard Deptartment \\ Datang Mobile Communications Equipment Co., Ltd. \\ Beijing, China \\ gaoqiubin@datangmobile.cn
}

\begin{abstract}
The multiple antenna technique is one of the most promising technologies to enhance system performance to reach the challenging requirements of IMT-Advanced technical requirement ITU-R M.2134 ${ }^{[1]}$. Channel state information is essential to enable closed-loop multiple antenna techniques. Channel reciprocity is an efficient and economic way of providing network with channel state information. In this paper, utilization of channel reciprocity in advanced MIMO system is discussed. Advantages and drawbacks of channel reciprocity are both discussed in detail. Simulation results are also provided to demonstrate efficiency of channel reciprocity.
\end{abstract}

Keywords: channel reciprocity, beamforming, multiple antennas

\section{Introduction}

Multiple antennas techniques are widely adopted by various standardization bodies, such as WiMax and 3GPP LTE. The challenging requirement of IMT-Advanced ${ }^{[1]}$ necessitates even more complicated multiple antennas techniques, e.g., multiple user MIMO (MU-MIMO), coordinated multiple points transmission / reception (CoMP), etc. Channel state information (CSI) at network side is indispensable to fully exploit the potential of such complex multiple antennas techniques. In FDD system, user equipment (UE) provides network side with quantized CSI through feedback channel. Evidently, it will occupy a large portion of uplink capacity. In TDD system, channel reciprocity can be used to reduce the feedback overhead. It is a promising direction of advanced MIMO techniques.

In this paper, the principle of channel reciprocity is first briefly introduced in section II. Single stream and dual-stream beamforming based on channel reciprocity are described in section III and section IV, respectively. Evolved beamforming techniques in LTE Rel-10 and beyond are envisioned in section V. Section VI and section VII discuss channel reciprocity in FDD and realistic considerations. Section VIII presents numerical results of several transmission schemes. Finally, conclusions are drawn in section IX.

\section{Principles of channel reciprocity}

When perfect channel state information (CSI) is available at the transmitter, linear precoding can be used to increase spectrum efficiency or enhance link reliability. The optimal linear precoder that achieves the channel capacity was shown to be the cascade of a beamforming matrix and a power allocation matrix ${ }^{[3]}$. The beamforming matrix consists of the right singular vectors of the channel matrix, while the power allocation matrix is obtained by water-filling over eigenmodes corresponding to the non-zero singular values.
Assumption of perfect CSI at the transmitter is often unrealistic. In some practical systems, CSI is sent to the transmitter through a finite rate feedback channel. The receiver usually selects the best precoder from a codebook, which is designed in advance and stored at both transmitter and receiver. The index of the selected precoder (quantized precoder) is then sent to the transmitter through the feedback channel. The feedback would occupy a certain portion of the scarce uplink resources, which decreases spectrum efficiency of uplink transmission. Since the capacity of uplink channel is limited, quantization error exists inevitably. Quantization error would degrade the gain of closed loop linear precoding.

Fortunately, in a TDD system, uplink and downlink channel is reciprocal, i.e., channel reciprocity holds. Channel reciprocity comes from the fact that propagation of electromagnetic wave is reversible, i.e., if electromagnetic wave is arriving at point $\boldsymbol{B}$ from point $\boldsymbol{A}$ through a specific path, the electromagnetic wave emitted at point $\boldsymbol{B}$ can arrive at point $\boldsymbol{A}$ through the same path. The same traveling path suggests that path attenuation, delay and phase offset are the same. In a wireless communication system, this implies that the channel from $\boldsymbol{A}$ to $\boldsymbol{B}$ is the same to the channel from $\boldsymbol{B}$ to $\boldsymbol{A}$. To be specific, in a cellular system, the uplink channel and the downlink channel are the same ${ }^{1}$. This phenomenon is depicted in Figure 1.
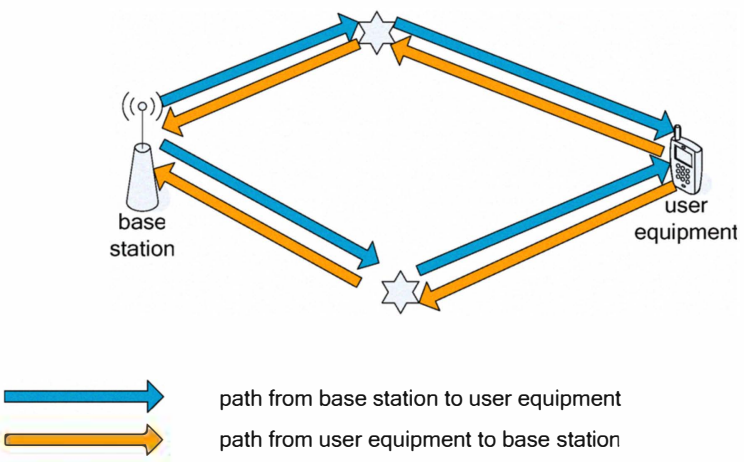

Figure 1: Description of channel reciprocity

To obtain uplink CSI, UEs are scheduled to transmit pilot signal at some time and frequency tones. Base station then estimates the uplink channel via the pilot signals. Denote the estimated uplink channel as $\boldsymbol{H}_{U L}$, an $\boldsymbol{M} \times \boldsymbol{N}$ matrix. $\boldsymbol{M}$ and $\boldsymbol{N}$ are number of antennas at base station and UE, respectively. Due to channel reciprocity, downlink channel can be obtained directly from uplink channel, i.e., $\boldsymbol{H}_{D L}=\boldsymbol{H}_{U L}^{T}$, where $\boldsymbol{A}^{T}$ is the transpose of matrix A.

\footnotetext{
${ }^{1}$ The link from base station to UE is called downlink and the link from UE to base station is called uplink.
} 
In commercial wireless communication systems, such as LTE, the uplink pilot signal is called sounding reference signal (SRS). SRS signal is transmitted periodically over bandwidth indicated by base station.

\section{Beamforming in LTE Rel-8}

Beamforming is a technology targeting improving coverage, capacity and reducing interference in systems equipped with closely spaced antenna array. It is successfully used in TDSCDMA. In LTE Rel-8, it is also standardized as an important multiple antenna technique to enhance cell edge throughput. Therefore, we take beamforming in LTE as an example to illustrate the usage of channel reciprocity. The procedure is illustrated in Figure 2.

As mentioned above, SRS signal is transmitted every few subframes. Base station estimates the uplink channel in those subframes and converts it to downlink channel. We denote the obtained downlink channel on subcarrier $k$ as $\boldsymbol{H}_{\boldsymbol{k}}$, then the beamforming weight can be calculated by eigen vector based beamforming (EBB) or by grid of beams (GoB). We will explain these two algorithms in the following separately.

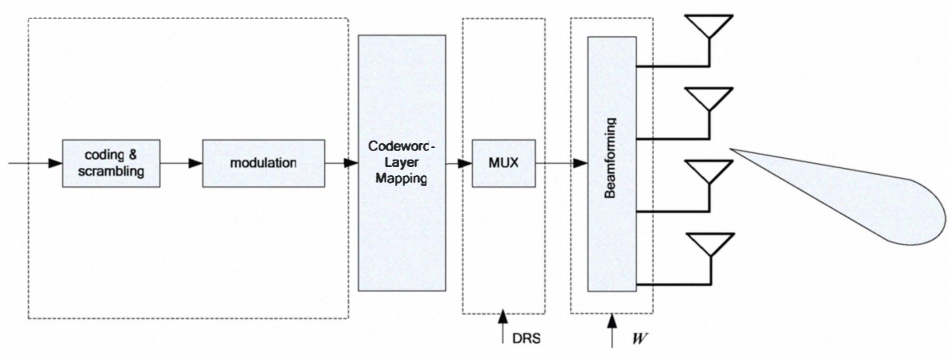

Figure 2: Illustration of beamforming

\section{A. $\quad$ EBB}

Assuming that UE employs maximum ratio combining (MRC) receiver, then the output SNR can be written as

$$
\text { SNR }=\frac{W^{H} H_{k}^{H} H_{k} W}{\sigma^{2}}
$$

where $\boldsymbol{W}$ is the beamforming weight on subcarrier $k$, and $\boldsymbol{\sigma}^{\mathbf{2}}$ is the variance of noise interference. Beamforming weight of EBB is designed by maximizing the output SNR:

$$
W=\operatorname{argmax} \mathrm{SNR}=\underset{\|W\|=1}{\operatorname{argmax}} \frac{W^{H} H_{k}^{H} H_{k} W}{\sigma^{2}}
$$

It is well known that solution to the above optimization problem is the principal right singular vector of $\boldsymbol{H}_{\boldsymbol{k}}$. The beamforming weight within a physical resource block (PRB) is better to be common for all subcarriers, since the channel for demodulation is derived by interpolation. A straightforward generalization of the maximum output SNR criterion is maximization of the average output SNR over a PRB:

$$
\mathrm{SNR}=\frac{1}{K} \sum_{k=1}^{K} \frac{W^{H} H_{k}^{H} H_{k} W}{\sigma^{2}}=\frac{W^{H}\left(\sum_{k=1}^{K} H_{k}^{H} H_{k} / K\right) W}{\sigma^{2}}
$$

$$
\boldsymbol{R}=\sum_{k=1}^{K} \boldsymbol{H}_{k}^{H} \boldsymbol{H}_{k} / K
$$

It is easily verified that the beamforming weight is given by

$$
W=\underset{\|W\|=1}{\arg \max } W^{H} R W
$$

Again, the optimal beamforming weight is the principal eigen vector of $\boldsymbol{R}$. The covariance matrix can also be averaged over larger bandwidth than a PRB, e.g., the whole bandwidth.

\section{B. GOB}

The spatial domain is divided into $\boldsymbol{L}$ subsectors; each subsector is represented by the antenna response corresponding to the central direction of the subsector. Denote the $l^{\text {th }}$ steering vector as

$$
v_{l}=\left[\begin{array}{c}
1 \\
e^{-j 2 \pi \frac{d}{\lambda} \sin \left(\theta_{l}\right)} \\
\vdots \\
e^{-j 2 \pi \frac{(N-1) d}{\lambda} \sin \left(\theta_{l}\right)}
\end{array}\right]
$$

where $\boldsymbol{d}$ is the distance between adjacent antenna elements, $\boldsymbol{\lambda}$ represents wavelength. The beamforming weight is then found by

$$
W=\underset{v_{l}, l=1, \ldots, L}{\arg \max } v_{l}^{H} R v_{l}
$$

where $\boldsymbol{R}$ is the wideband or subband covariance matrix. From the above formulation, it can be recognized that beamforming weight of $\mathrm{GoB}$ is derived based on the direction of arrival (DoA). Since DoA is rather stable over frequency and time domain, GoB algorithm is robust to channel estimation error, sounding delay, precoding granularity etc. Besides, DoA is also reciprocal in FDD system, i.e., GoB algorithm is applicable for FDD.

In order to facilitate link adaptation, base station should also have the knowledge of channel quality information (CQI). Since UE does not know the beamforming weight, it is impossible for use equipment to calculate CQI accurately. In LTE, UE may be configured to feed back a CQI with an assumption that the transmission scheme is transmit diversity. Upon receiving the CQI reported by UE, base station modified it according to beamforming gain. This is because beamforming gain is a relatively stable value.

For purpose of demodulation, dedicated reference signal is transmitted together with data. The reference signal is weighted by the same weight as data. Therefore, channel estimated by UE from reference signal is a composite of the channel and beamforming weight. UE can use it directly to demodulate data without any additional information about the beamforming weight.

\section{Dual-stream beamforming in LTE Rel-9}

In rich scattering environment, closely spaced antenna array may also support multiple-stream transmission. Particularly, if crosspolarized antenna (or group antenna) is employed, the probability of multiple-stream transmission is greatly increased. Therefore, LTE Rel-9 standardized dual-stream beamforming, which is illustrated in Figure 3. Dual-stream beamforming can be realized with or without precoding matrix indicator (PMI) report.

Define the covariance matrix as 


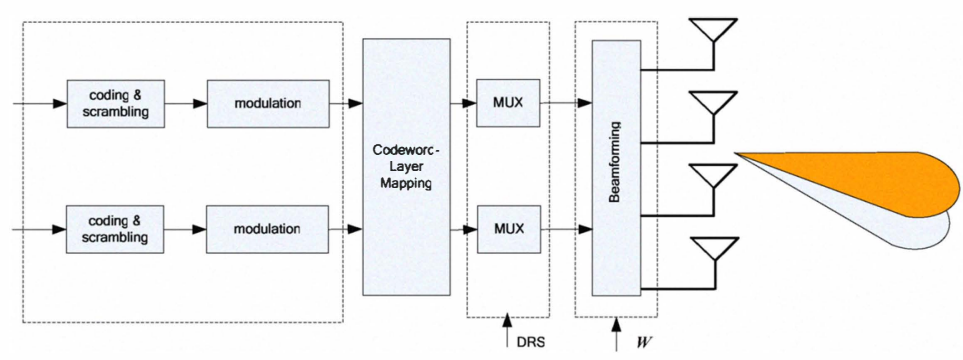

Figure 3: Illustration of dual-stream beamforming

\section{A. Without PMI report}

If dual-stream beamforming is configured without PMI/RI reporting, generating of beamforming weight is solely based on channel reciprocity. Similar to single stream beamforming, the beamforming weight can be calculated via EBB algorithm. Since two streams are transmitted, the first two principal eigenvectors are adopted as the beamforming weight.

As mentioned above, a typical application scenario of dual-stream beamforming is closely-spaced cross-polarized (or group antenna) antenna array. An important characteristic of such antenna array is that the antennas can be divided into two groups based on their polarization and that the channels within a group often tend to be rather correlated. On the other hand, channels belonging to two different groups correspond to orthogonal polarizations and are thus typically uncorrelated. In this scenario, the beamforming weight can be construct as

$$
W=\left[\begin{array}{ll}
a & 0 \\
0 & a
\end{array}\right] U
$$

where $\boldsymbol{a}$ is beamforming weight for the correlated part, and can be determined by EBB or GoB algorithms. $\boldsymbol{U}$ is a matrix with orthogonal columns, e.g., can be selected as scaled identity matrix. The beamforming weight is divided into two parts, $\boldsymbol{a}$ is used to take care of the correlated parts of the channel, and $\boldsymbol{U}$ is used to combine the two uncorrelated parts.

The same as single stream beamforming, a CQI with an assumption of transmit diversity is reported. Base station computes CQI for two transmission codewords according to the reported CQI and the ratio between the first two eigenvalues of the covariance matrix $\lambda_{2} / \lambda_{1}$, i.e.,

$$
\begin{aligned}
& \mathrm{CQI}_{1}=\mathrm{CQI} \times \boldsymbol{G}_{\boldsymbol{B} F} / \mathbf{2} \\
& \mathrm{CQI}_{2}=\mathrm{CQI}_{1} \times \boldsymbol{\lambda}_{\mathbf{2}} / \boldsymbol{\lambda}_{1}
\end{aligned}
$$

where CQI is reported by $\mathrm{UE}, \mathrm{CQI}_{\mathrm{i}}$ represents CQI for the $i^{\text {th }}$ stream, $\boldsymbol{G}_{\boldsymbol{B} \boldsymbol{F}}$ is the beamforming gain.

\section{B. With PMI report}

As an alternative, dual-stream beamforming can also be realized with assistant of PMI/RI report. Assume that two cell-specific reference signal (CRS) ports are transmitted from two antenna groups individually. The CRS ports are assumed to be weighted by a beamforming weight, which generates a wide beam, say $\boldsymbol{b}$. UE estimate the composite channel through the CRS port

$$
\boldsymbol{H}_{\text {eff }}=\boldsymbol{H}\left[\begin{array}{ll}
\boldsymbol{b} & \mathbf{0} \\
\mathbf{0} & \boldsymbol{b}
\end{array}\right]
$$

RI and PMI are selected based on $\boldsymbol{H}_{\text {eff }}$, and they are reported. Denote the precoding matrix indicated by the reported PMI by $\boldsymbol{V}$. Then the beamforming weight is given by

$$
W=\left[\begin{array}{ll}
a & 0 \\
0 & a
\end{array}\right] V
$$

where $\boldsymbol{a}$ is obtained through channel reciprocity, and can be determined via either EBB or GoB algorithms. Off course, $\boldsymbol{a}$ can be equal to $\boldsymbol{b}$. If $\boldsymbol{a} \neq \boldsymbol{b}$, there is a mismatch between the desired precoder and the transmission precoder. Since demodulation relies on dedicated reference signal, the mismatch cause minors performance degradation.

$\mathrm{CQI}$ is reported together with the reported PMI/RI. If $\mathrm{RI}=2$, one CQI for each stream is reported, otherwise, only one CQI is reported and tranmission may degenerated to single stream beamforming. The reported CQI is calculated based on assumption of closed-loop spatial multiplexing.

\section{Channel reciprocity in LTE Rel-10 and beyond}

LTE-A system targets at peak rates greater than $1 \mathrm{Gbps}$ over bandwidth $100 \mathrm{MHz}$ with low mobility. In order to meet the requirements, unto 8 layers transmission is considered. Dual-stream stream can be directly extended to multiple-stream transmission. The principle is the same as dual-stream beamforming, hence we do not address it much in this paper.

Coordinated multiple point transmission/reception (CoMP) is another technique considered in LTE-A to improve spectral efficiency and coverage of high data rates in both low load and high load scenarios. CoMP implies dynamic coordination among multiple geographically separated transmission points. For example, multiple cells coordinate time/frequency resources allocated to UEs and the beam direction toward UE to reduce interference to each other (CS/CB). More aggressively, multiple cells transmit data toward a UE simultaneously (JP). Coherently or noncoherently combining of signal transmitted from multiple cells improves the quality of signal received by UE. Since channel among different cells are typically independent, spatial diversity or spatial multiplexing is also possible. Examples of CoMP transmission scheme are show in Figure 4.

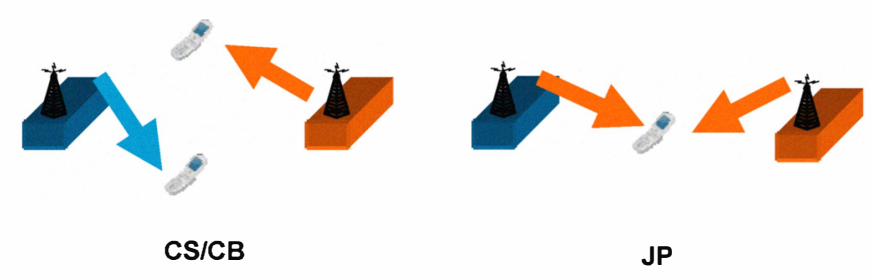

Figure 4: CoMP transmission scheme

In order to implement closed-loop precoding at network side, CSI is still required. The requirement is more stringent than single cell transmission, since CSI related to multiple links (between multiple transmission points and UE) are needed. Taking into account the special transmission scheme, CSI should be more accurate, implying that more feedback overhead is needed. In a word, the feedback overhead is much larger than ever before. For example, quantized channel matrix or covariance matrix may be needed, translating into overhead of almost $50 \%$ of uplink capacity ${ }^{[4]}$. If 
channel reciprocity can be exploited, is will be very competing, since no extra feedback overhead is needed.

SRS signal sent by a UE should be able to arrive at every transmission point. Since the transmission points are geometrically separated, some of them may be much further than others. Transmission point of serving cell is usually the nearest to UE. This means that signal strength of SRS signal at some point may be much weaker than that of serving cell. Besides, SRS or data signal scheduled by the target point (cell) may serve as an interference. The consequence is that the effective SINR of SRS signal at some points may be rather small. Therefore, channel estimation accuracy can not be guaranteed, which will certainly impair performance of CoMP transmission. Two method may be adopted to solve the problem: a) coordinating transmission of SRS signal in different cell to ensure orthogonality; b) promoting transmission power of SRS signal.

\section{Channel reciprocity in FDD}

The distance between the uplink and downlink frequency band in FDD system is usually large. Thus, channel reciprocity does not hold in FDD system in strict sense. However, regarding the statistical channel information, it is well known that the (long-term wideband) channel covariance matrix changes much more slowly than the coherence time and bandwidth of the channel. That is, if the duplex distance is sufficiently small relative to carrier frequency, a covariance matrix estimated over an uplink frequency band, is valid also on a downlink frequency band. Moreover, frequency translation techniques can be used to improve the accuracy. An example of such techniques was proposed in [4], and is summarized below. The uplink and downlink antenna responses (for a uniform linear array)

$$
a_{U L}(\theta)=\left[\begin{array}{c}
1 \\
e^{-j 2 \pi \frac{d f_{U L}}{\lambda f_{0}} \sin (\theta)} \\
\vdots \\
e^{-j 2 \pi \frac{d}{\lambda} \frac{f_{U L}}{f_{0}}(N-1) \sin (\theta)}
\end{array}\right]
$$

and

$$
a_{D L}(\theta)=\left[\begin{array}{c}
1 \\
e^{-j 2 \pi \frac{d f_{D L}}{\lambda f_{0}} \sin (\theta)} \\
\vdots \\
e^{-j 2 \pi \frac{d f_{D L}}{\lambda f_{0}}(N-1) \sin (\theta)}
\end{array}\right]
$$

are related as

$$
a_{D L}(\theta)=T(\theta) a_{U L}(\theta)
$$

where the translation matrix is given by

$$
T(\theta)=\operatorname{diag}\left(1, e^{j 2 \pi \frac{d f_{U L}-f_{D L}}{\lambda} f_{0} \sin (\theta)}, \ldots, e^{j 2 \pi \frac{d f_{U L}-f_{D L}}{\lambda} f_{0}(N-1) \sin (\theta)}\right)
$$

and $f_{0}$ is the carrier frequency that the ULA is designed for, $\boldsymbol{d}$ is the antenna spacing, $\boldsymbol{\lambda}$ is wavelength corresponding to $f_{\mathbf{0}}, \boldsymbol{\theta}$ is the angle of arrival/departure, and $f_{D L}$ and $f_{U L}$ are the downlink and uplink carrier frequencies respectively.

By estimating the dominating direction of arrival (DOA) in the uplink, $\boldsymbol{\theta}_{\mathbf{0}}$, an improved downlink covariance estimate may be obtained as

$$
\hat{R}_{D L}=T\left(\theta_{0}\right) R_{U L} T^{H}\left(\theta_{0}\right)
$$

With the estimated downlink covariance matrix $\hat{\boldsymbol{R}}_{\boldsymbol{D} L}$, both single and dual-stream beamforming can be conducted. Furthermore, coordination between multiple cells is also possible with such information.

Note that, for closely spaced antenna array, the above translation is rather accurate. However, for diversity antennas, the performance degradation is not negligible.

\section{Realistic considerations}

In above discussions, we assume strict channel reciprocity holds. However, even in TDD system, channel reciprocity holds for only the physical propagation channel. Hence, whenever there is a noticeable difference between the transfer characteristics of various analog parts used at TX/RX, there is no reason to assume reciprocity of these variations at $\mathrm{TX} / \mathrm{RX}$ and therefore reciprocity of the equivalent channel.

Hence, it is important to understand the magnitude of variations observed at different analog parts and their influence on the accuracy of the reciprocity assumption when applied to the equivalent channel. Moreover, in antenna array systems, each antenna will have it is own transmitter/receiver chains which are not necessarily the same for all antennas. Therefore, antenna array calibration is required.

Since RF chain is valuable, UE is usually equipped with less transmitting RF chains than receiving RF chains. In this case, SRS signal can be transmitted in turn, that is, a switch is needed. Sometimes, a switch does not even exist, base station can only obtain partial CSI via channel reciprocity. Dual-stream beamforming is still possible. An example is to use the principal eigenvector as the beamforming weight for the first stream, beamforming weight for the second stream can be selected as a random vector that is orthogonal to the beamforming weight of the first stream. Another method is to use directly first two principal eigenvectors as the beamforming weight. Although partial CSI is available, performance is degraded and the application will be limited to low rank transmission.

Another source of non-reciprocity regards interference. Interference of uplink comes from UEs, and interference of downlink comes from base stations, hence they are different. Base on the CSI obtained from channel reciprocity, base station can adapt the direction of signal toward the actual channel. Link adaptation is not feasible without information regarding interference. That is why UE should feed back CQI for single and dual-stream beamforming. The reported CQI reflects downlink interference experienced by the UE.

Utilization of channel reciprocity relies on SRS signal or other uplink signal. Off course, they can not be transmitted all the time. They must be sent with some interval between two successive transmission opportunities. This implies that, the used CSI obtained via channel reciprocity may be expired if the interval exceeds channel coherence time. Therefore, the period of uplink reference signal should be selected appropriately according the Doppler spread.

\section{Numerical evaluations}

In this section we give some evaluation results to demonstrate the efficiency of channel reciprocity. 


\section{A. Dual-stream beamforming}

In this section, we evaluate the performance of single and dualstream beamforming by system simulations. Simulation parameters are listed in Table I. The spectral efficiency for three transmission schemes is shown in Table II.

Table I: Simulation parameters for dual-stream beamforming

\begin{tabular}{|l|l|}
\hline Parameters & Values \\
\hline Scenario & 3GPP Case 1-2D, high spread \\
\hline Beamforming algorithm & EBB \\
\hline Precoding granularity & $1 \mathrm{PRB}$ \\
\hline Channel estimation & Perfect \\
\hline Sounding signal period & $5 \mathrm{~ms}$ \\
\hline BS antenna configuration & $4+4$ polarized, 0.5 wavelength \\
\hline UE antenna configuration & $1+1$ polarized, 0.5 wavelength \\
\hline
\end{tabular}

Table II: Spectral efficiency of beam forming

\begin{tabular}{|l|c|c|}
\hline & $\begin{array}{c}\text { Cell average } \\
\text { spectrum } \\
\text { efficiency } \\
{[\mathrm{bps} / \mathrm{Hz}]}\end{array}$ & $\begin{array}{c}\text { 5\% cell edge } \\
\text { throughput } \\
{[\mathrm{bps} / \mathrm{Hz} / \mathrm{user}]}\end{array}$ \\
\hline Single-stream & 2.08 & 0.089 \\
\hline SU-Dual-stream & 2.40 & 0.071 \\
\hline $\begin{array}{l}\text { SU-Single/dual } \\
\text { adaptive }\end{array}$ & 2.41 & 0.092 \\
\hline
\end{tabular}

Several observations can be made from the above simulation results:

- SU-Dual-stream beamforming provides about $15 \%$ gain in terms of cell average spectrum efficiency over single stream beamforming. There is about $20 \%$ loss in terms of cell edge throughput. The intuition is that, geometry of cell edge UE is low, and hence can not support dual-stream transmission.

- Adaptive single/dual stream beamforming means that the rank is determined based on geometry of UE. By this way, cell center UE is more likely to use dual-stream beamforming, while cell edge UE is allowed to use single-stream beamforming. Adaptive beamforming provide about $15 \%$ gain on cell average spectrum efficiency without decreasing cell edge throughput.

\section{B. CoMP}

In this section, we evaluate the performance of $\mathrm{CS} / \mathrm{CB}$, which is a prevalent kind of CoMP transmission. Simulation parameters are listed in Table III. The results are shown in Table IV and Table V. System-wide CS/CB is that the coordination is carried out throughout the network (57 sectors in this simulation), while intrasite coordination is limited to 3 co-located sectors.

Table III: Simulation parameters for CoMP

\begin{tabular}{|l|l|}
\hline Parameters & Values \\
\hline Scenario & 3GPP Case 1-2D, high spread \\
\hline Beamforming algorithm & EBB \\
\hline Precoding granularity & 1 PRB \\
\hline Channel estimation & Perfect \\
\hline Sounding signal period & 5ms \\
\hline BS antenna configuration & 4Tx ULA, 0.5 wavelength \\
\hline UE antenna configuration & 2Rx ULA, 0.5 wavelength \\
\hline
\end{tabular}

Table IV: Performance of CS/CB with SU-MIMO

\begin{tabular}{|l|c|c|}
\hline & $\begin{array}{c}\text { Cell average } \\
\text { spectrum } \\
\text { efficiency [bps/Hz] }\end{array}$ & $\begin{array}{c}5 \% \text { cell edge } \\
\text { throughput } \\
\text { [bps/Hz/user] }\end{array}$ \\
\hline No CS/CB & 2.03 & 0.096 \\
\hline Intra-site CS/CB & 2.12 & 0.095 \\
\hline System-wide CS/CB & 2.26 & 0.111 \\
\hline
\end{tabular}

Table V: Performance of CS/CB with MU-MIMO

\begin{tabular}{|l|c|c|}
\hline & $\begin{array}{c}\text { Cell average } \\
\text { spectrum } \\
\text { efficiency }[\mathrm{bps} / \mathrm{Hz}]\end{array}$ & $\begin{array}{c}5 \% \text { cell edge } \\
\text { throughput } \\
{[\mathrm{bps} / \mathrm{Hz} / \mathrm{user}]}\end{array}$ \\
\hline No CS/CB & 2.86 & 0.120 \\
\hline Intra-site CS/CB & 3.04 & 0.124 \\
\hline System-wide CS/CB & 3.10 & 0.133 \\
\hline
\end{tabular}

Several observations can be made from the above simulation results:

- MU-MIMO transmission based on channel reciprocity gives over $40 \%$ gain on average spectrum efficiency, and over $20 \%$ gain on cell edge throughput.

- Intra-site coordination provides marginal gain.

- System-wide coordination provides about $10 \%$ gain.

\section{Discussion and conclusions}

In this paper, we reviewed the utilization of channel reciprocity in various MIMO transmission schemes, including single/dual-stream beamforming, CoMP, etc. Advantages of channel reciprocity over feedback include:

- Accuracy of CSI derived form channel reciprocity is higher than that of feedback;

- Feedback overhead is reduced by utilizing channel reciprocity.

Despite the promising aspects, channel reciprocity suffers from some non-ideal factors, such RF misalignment, non-reciprocity of interference, etc. How to overcome these problems needs further study. Finally, some numerical results are given to demonstrate the efficiency of channel reciprocity.

\section{References}

[1] ITU-R Report M.2134, Requirements related to technical performance for IMT-Advanced radio interface(s), 2008.

[2] 3GPP TR 36.814, "Further advancements for E-UTRA physical layer aspects"

[3] I. E. Telatar, "Capacity of multiantenna Gaussian channels," Eur. Trans. Telecommun., vol. 10, no. 6, pp. 585-595, Nov. 1999.

[4] 3GPP R1-092737, "On CSI feedback for IMT-Advanced Fulfilling CoMP Schemes," Ericsson, ST-Ericsson 